\title{
@ Clipping of bilateral MCA aneurysms and a coiled ACOM aneurysm through a modified lateral supraorbital craniotomy
}

Ziad A. Hage, M.D. and Fady T. Charbel, M.D.

Department of Neurosurgery, University of Illinois at Chicago

We showcase the microsurgical clipping of a left middle cerebral artery (MCA) aneurysm-(B) done through a modified right lateral supraorbital craniotomy, as well as clipping of a previously coiled anterior communicating (ACOM) artery aneurysm-(C) and a bilobed right MCA aneurysm-(A). Splitting of the right sylvian fissure is initially performed following which a subfrontal approach is used to expose and dissect the contralateral sylvian fissure. The left MCA aneurysm is identified and clipped. The ACOM aneurysm is then clipped following multiple clip repositioning based on flow measurements. The right MCA aneurysm is then identified and each lobe is clipped separately.

The first picture showcased in this video is a side to side right and left ICA injection in AP projection. In this picture, $(\mathrm{A})$ points to the bilobed right MCA aneurysm, $(B)$ to the left middle cerebral artery (MCA) aneurysm, and (C) to the previously coiled anterior communicating (ACOM) artery aneurysm. The red dotted line shows that both MCA aneurysms lie within the same plane which makes it easier to clip both of them, through one small craniotomy.

The video can be found here: http://youtu.be/4cQC7nHsL5I.

KEY WORDS aneurysm clipping; lateral supraorbital craniotomy; flow measurement 